\section{Cahiers de Narratologie}

Analyse et théorie narratives

$31 \mid 2016$

Sérialité narrative. Enjeux esthétiques et économiques

\title{
Les enjeux sociaux d'une narration sérielle fictive : Little Sammy Sneeze de Winsor McCay (1904-1906)
}

\section{Françoise Revaz}

\section{(2) OpenEdition}

\section{Journals}

\section{Electronic version}

URL: http://journals.openedition.org/narratologie/7593

DOI: $10.4000 /$ narratologie. 7593

ISSN: 1765-307X

Publisher

LIRCES

\section{Electronic reference}

Françoise Revaz, « Les enjeux sociaux d'une narration sérielle fictive : Little Sammy Sneeze de Winsor McCay (1904-1906) », Cahiers de Narratologie [Online], 31 | 2016, Online since 22 December 2016, connection on 15 November 2019. URL : http://journals.openedition.org/narratologie/7593; DOI : 10.4000/narratologie.7593

This text was automatically generated on 15 November 2019.

Article L.111-1 du Code de la propriété intellectuelle. 


\title{
Les enjeux sociaux d'une narration sérielle fictive : Little Sammy Sneeze de Winsor McCay (1904-1906)
}

\author{
Françoise Revaz
}

1 La littérature d'imagination est réputée avoir pour principal enjeu le divertissement du lecteur (elle serait le lieu par excellence du plaisir « désintéressé » et ses effets sociaux seraient cantonnés à la sphère esthétique), alors même que la littérature dite «factuelle» (récits d'historiens, faits divers journalistiques ou reportages télévisés) aurait pour finalité la transmission de savoirs sur le monde.

2 L'objectif de cet article est de questionner cette idée reçue d'une fracture entre deux types de littératures, l'une " gratuite ", produite pour la seule distraction du lecteur, l'autre "utile», proposant une réflexion sur la société et visant ainsi à affiner la compréhension sociale du monde. Pour montrer que cette dichotomie est par trop caricaturale, j'ai choisi de me pencher sur un genre prototypique de la littérature d'imagination, dans sa forme la plus commerciale, à savoir la série de bandes dessinées. Le propre de cette littérature, généralement catégorisée comme " populaire » et donc dévalorisée, serait de favoriser la fonction économique au détriment de la fonction esthétique, l'a priori étant que la narration sérielle bédéique ne proposerait qu'une succession d'aventures au suspense intense et que l'intérêt du lecteur ne proviendrait que de l'attente impatiente du dénouement de l'intrigue ${ }^{1}$. Ce serait même ce seul ressort narratif $\mathrm{du}$ "suspense » qui aurait fidélisé le lectorat des publications sérielles, au début $\mathrm{du} \mathrm{XX}^{\mathrm{e}}$ siècle. De fait, le principe de la série est de proposer, selon une périodicité régulière, une nouvelle aventure mettant en scène le même héros et reproduisant invariablement le même schéma narratif. La fidélisation du public tient ainsi au plaisir qu'il a à retrouver du "même ", à savoir un univers diégétique dont il connaît déjà les règles et qui est habité par des personnages (le héros et ses éventuels comparses) dont le caractère, les habitudes et le type de comportement lui sont familiers. 


\section{Little Sammy Sneeze}

3 Les quelques exemples qui vont illustrer cet article sont tirés d'une série de bandes dessinées très populaire aux Etats-Unis dans les années 1900: la série Little Sammy Sneeze du célèbre Winsor McCay, illustrateur de presse, caricaturiste politique et, surtout, pionnier de la bande dessinée et du film d'animation ${ }^{2}$. Cette série est parue au rythme d'une planche hebdomadaire dans le New York Herald entre le 24 juillet 1904 et le 9 décembre 1906. Son mode de composition obéit à celui du stop comic: une courte histoire dont le contenu narratif s'articule autour d'une chute finale en forme de gag. Chaque épisode hebdomadaire met ainsi en scène un personnage récurrent, le " petit Sammy " mentionné dans le titre, et reproduit le même canevas narratif. En une séquence de six cases au cadrage strictement identique, McCay nous donne à voir toujours le même déroulement événementiel : dans les cinq premières cases, les phases successives de l'éternuement irrépressible du petit Sammy (accompagnées d'onomatopées qui correspondent à chaque étape de l'ouverture de la bouche ${ }^{3}$ ) jusqu'à la déflagration nasale qui, dans la cinquième case, vient bouleverser le monde environnant ${ }^{4}$. Quant à la sixième et dernière case, elle montre systématiquement Sammy expulsé par un coup de pied au derrière magistral, dispensé par un adulte. En somme, l'intrigue est minimale 5 .

4 On soulignera que l'éternuement de Sammy est totalement indépendant de sa volonté, comme le précisent les deux sous-titres de la série (" Il ne pouvait pas s'en empêcher », Il ne le sentait jamais venir $\left.{ }^{6} ! »\right)$. En outre, l'enfant n'entre jamais en interaction avec son environnement. Il ne parle pas et occupe une position de spectateur (de nombreuses planches sont consacrées à la représentation d'un spectacle auquel il assiste). Il est totalement impassible et ne semble pas du tout affecté par son éternuement. On remarque d'ailleurs que, lors de la "déflagration », sa position reste identique à celle qu'il occupe dans les cases précédentes, alors même que tout ce qui l'entoure est totalement bouleversé. Quant à la destruction qu'occasionne son éternuement, elle est disproportionnée, voire, dans certaines planches, quasiment cataclysmique. Obéissant au principe de répétition/variation des stop comics, l'éternuement de Sammy est reproduit à l'identique d'un épisode à l'autre, tant dans les différentes phases du mouvement de la bouche que dans les onomatopées qui les accompagnent, tandis que le lieu dans lequel il se produit est à chaque fois différent, tout comme les personnages qui entourent Sammy. Au fil de la série, un événement identique, l'éternuement, joue le rôle d'élément perturbateur, ce qui garantit la narrativité de la planche.

5 On notera enfin que les protagonistes de la série, les adultes qui s'activent autour de Sammy, sont toujours occupés à des actions stéréotypées, qu'il s'agisse d'une activité quotidienne banale (partager un repas), de la pratique d'un métier (horloger) ou d'un loisir (jeu de boules), ou encore d'une réunion mondaine (concert) ou d'un rituel (office religieux). La présence de ces scripts sociaux est importante dans la mesure où les phases associées à chaque script offrent au lecteur des balises importantes pour la compréhension de la séquentialité de la planche ${ }^{7}$.

6 La série Little Sammy Sneeze montre que la visée simplement récréative de ses anecdotes plaisantes se double résolument d'une visée sociale, voire politique, en offrant au public un regard critique sur le mode de vie de la classe moyenne américaine de l'époque. 


\section{Une série intrigante}

7 Même si le scénario est immuable (Sammy éternue et la phase explosive de son éternuement bouleverse le monde qui l'entoure), en termes d'attentes de la part du lecteur, il y a dans chaque épisode une forme de suspense due au retard dans la monstration de l'explosion. En effet, l'éternuement se développe durant les quatre premières cases, ce qui conduit le lecteur à s'engager sur le mode du pronostic, défini par Baroni (2007) comme l'«interprétation "descendante" visant à anticiper le développement futur de la "fabula" »(11). Certes, la vision tabulaire permet en principe de saisir d'un premier coup d'œil l'intégralité du contenu de la planche et, éventuellement, de prendre connaissance du dénouement par avance, mais lors de la réception de la série dans la page dominicale, l'imposant format du journal atténuait la possibilité d'épuiser le contenu de la planche au premier regard. Benoît Peeters (1990) précise à ce propos :

Le vaste espace de la Sunday page du New York Herald (large d'environ quarante centimètres et haute de cinquante-six) devait obéir à une double contrainte, s'offrant à une découverte globale autant que graduelle. Assez spectaculaire pour accrocher l'œil de celui qui feuilletait le journal d'une main discrète, il devait s'avérer assez passionnant pour le retenir sitôt qu'il commençait à lire. (Peeters, 1990 : 33)

On peut donc supposer une lecture « pas à pas » des vignettes, qui mobilise une attitude interprétative sur le mode du pronostic. Notons que le pronostic ne porte pas sur ce qui va advenir dans une phase ultérieure du récit (le lecteur sait à quoi s'attendre sur ce point), mais sur la forme visuelle que prendra la figuration des effets de l'éternuement. C'est effectivement cet aspect qui attise la curiosité du lecteur et garantit chaque semaine l'intérêt de l'épisode. En somme, le lecteur sait ce qui va se passer, mais il ne sait pas comment. Le côté intriguant se déplace donc du «Quoi?» vers le «Comment?».

9 Enfin, on soulignera le potentiel d'« attraction» de la série, les planches de McCay tablant sur le plaisir sadique du lecteur qui va découvrir chaque semaine les effets dévastateurs de l'éternuement de Sammy sur des activités et des lieux familiers. De ce point de vue, on peut affirmer qu'il y a bien un aspect récréatif dans la série de Sammy Sneeze et que cet aspect a certainement concouru à son succès commercial. Mais si l'on en reste à ce niveau de l'analyse, on passe certainement à côté de ce que McCay a voulu faire dans le cadre de cette série. Intéressons-nous donc aux autres caractéristiques du corpus qui vont mettre en évidence la visée sociale et politique de l'écriture de McCay.

\section{Une série parodique}

10 Avec Little Sammy Sneeze, McCay propose manifestement une parodie des séries de bandes dessinées produites par ses contemporains qui, pour la plupart, mettent en scène des enfants qui reproduisent inlassablement le même type de bêtises ou de mauvaises plaisanteries, comme, par exemple, la série américaine The Katzenjammer Kids (Pim, Pam, Poum dans la traduction française) de Rudolph Dirks où les trois garnements rivalisent de créativité pour empoisonner la vie du Capitaine. McCay raille cette exploitation d'une même forme de gag et pousse le principe répétitif jusqu'à l'absurde en fondant son scénario sur un événement banal : un simple éternuement. 
Dans la mesure où éternuer consiste en une manifestation réflexe et non contrôlable, Sammy se voit ainsi cantonné dans un rôle passif. De plus, son éternuement étant non intentionnel, Sammy se démarque nettement des garnements des autres séries, qui se réjouissent de chaque méfait dont ils sont responsables.

11 Sur le plan formel, également, McCay parodie le principe répétitif en reproduisant chaque semaine le même dispositif d'un gaufrier de six cases, de même format et de même cadrage. Dans la plupart des épisodes, la position de Sammy reste identique de case en case, cette récurrence étant soulignée par l'invariance du point de vue et de l'échelle de plan. Quand on sait avec quelle inventivité et quelle virtuosité McCay dessine les planches de Little Nemo, variant la mise en page chaque semaine, on ne peut qu'être interpellé par le côté répétitif de Little Sammy. En somme, avec Sammy, McCay lutte contre les produits stéréotypés issus de la production industrielle de la BD des années 1900 en produisant lui-même une parodie de ces procédés.

\section{Une série satirique}

12 Une dernière caractéristique du corpus est le regard critique et moqueur que McCay porte sur la société de l'époque. En ce sens, la série Little Sammy Sneeze ne fait pas que satisfaire le plaisir du lecteur avec des anecdotes divertissantes. Au-delà du gag de l'éternuement, McCay propose une vision satirique des comportements de ses contemporains. Par exemple, dans chaque planche, les adultes de la classe moyenne sont représentés avec les attributs formels de la respectabilité : smokings et hauts-deforme pour les hommes et robes à corset pour les femmes. Souvent ces personnages sont très bavards et échangent des propos sans queue ni tête, ce qui contraste avec l'attitude de Sammy qui ne dit jamais rien. Observateur attentif des comportements humains, McCay rend compte de la réalité sociale du New York des années 1900 en montrant les activités humaines, les pratiques sociales, la psychologie et la langue des différentes classes de la société. Chaque planche hebdomadaire permet ainsi de s'interroger sur le mode de vie de la société américaine. 


\section{Quelques exemples...}

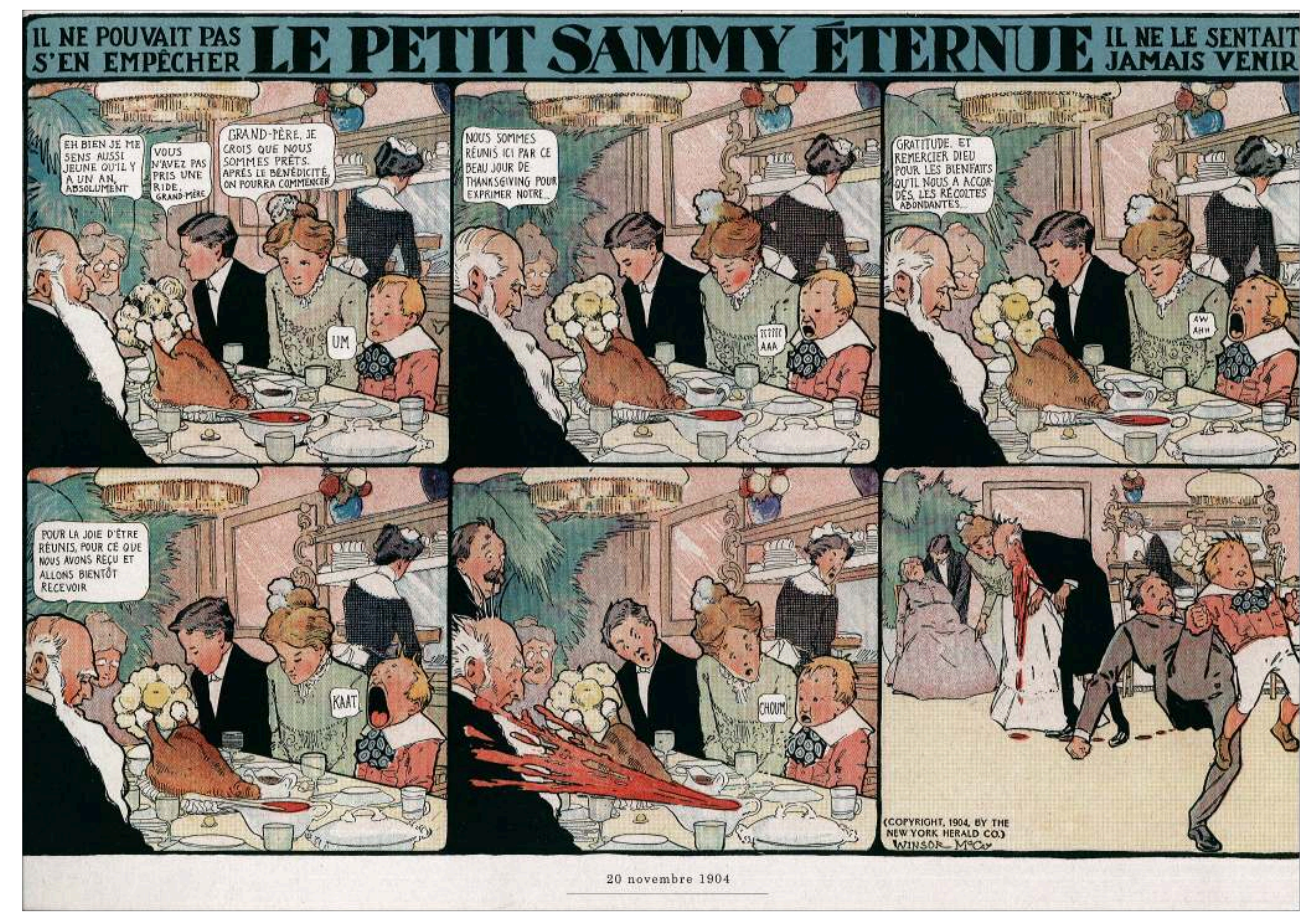

La planche 1 situe l'éternuement de Sammy lors de la fête de Thanksgiving qui réunit ses parents, ses grands-parents et quelques invités autour d'une table bien dressée sur laquelle trône la dinde traditionnelle. Le gag consiste ici dans le fait que le résultat de l'éternuement dévastateur (qui va envoyer le contenu de la saucière sur le plastron du grand-père) semble annoncé, dans la $4^{\mathrm{e}}$ case, par les paroles de ce dernier lorsqu'il évoque «ce que nous avons reçu et allons bientôt recevoir »! Mais un autre élément important à souligner, présent dans de nombreux épisodes, est la représentation du langage, langage dont McCay aime à montrer la banalité ou l'incongruité. A cet égard, on peut souligner d'une part la futilité de l'échange entre la grand-mère et le père de Sammy dans la $1^{\mathrm{e}}$ case, d'autre part le ridicule du benedicite récité par le grand-père, dans les cases 3 à 4 , tourné en dérision par McCay qui s'amuse à lui faire prédire la catastrophe finale. En fait, cette planche est représentative de l'habitude qu'a McCay de se moquer des rituels religieux, que ceux-ci se déroulent dans le cadre familial, comme ici avec la prière du grand-père, ou en société, à l'église par exemple, lors des offices. 


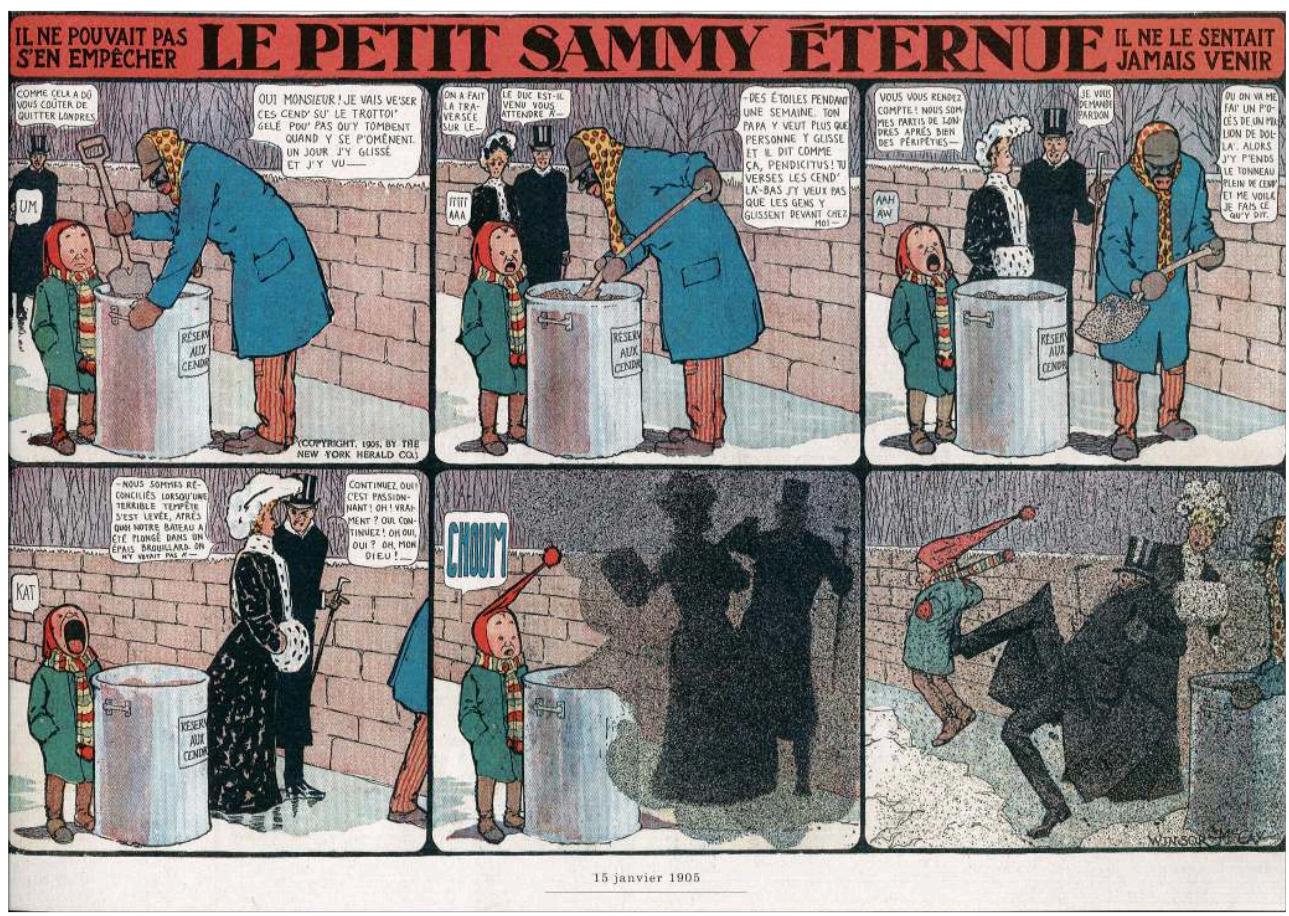

Dans cette deuxième planche, l'éternuement de Sammy advient en plein air, sur un trottoir gelé qu'un domestique noir a le devoir de recouvrir de cendres afin d'éviter la chute des passants. Au premier plan, on voit conjointement le développement de l'éternuement de Sammy et l'activité du domestique noir qui s'apprête à répandre les cendres. L'arrière-plan est également intéressant. On peut y observer la déambulation d'un couple de promeneurs, manifestement de classe sociale aisée, déambulation durant laquelle un récit mondain est proféré. Tout comme dans la planche précédente, l'inanité du langage verbal est moquée. Cette fois, c'est la futilité du récit de la dame élégante qui est mise en évidence. Son récit commence in medias res et " hors champ ", puisque, dans la $1^{\mathrm{e}}$ case, on ne voit pas encore la « narratrice » et que seule la réplique de l'homme coiffé d'un chapeau haut-de-forme laisse supposer que quelqu'un a commencé de lui parler. Le récit est dialogal ; il suscite des réactions de l'interlocuteur, qui pose des questions et émet des commentaires. Il avance ainsi par bribes. De plus, il est interrompu, dans la $4^{\mathrm{e}}$ case, au moment du nœud dans l'intrigue narrée («nous sommes réconciliés lorsqu'une terrible tempête s'est levée... »). Ce qui est remarquable, ici, c'est la performance narrative qui se voit disqualifiée et sabotée, puisqu'on ne saura rien de la façon dont s'est terminé le voyage en bateau. De plus, les réactions de l'interlocuteur à l'écoute du récit sont tournées en ridicule. Par exemple, dans la case 4, les exclamations «continuez, oui ! c'est passionnant! oh! vraiment? oui, continuez! oh oui, oui? oh, mon dieu! " paraissent pour le moins excessives. On notera enfin que le type de perturbation que va provoquer l'éternuement de Sammy, à savoir le nuage de cendres, est indirectement "annoncé » dans la $4^{\mathrm{e}}$ case par la réplique de la femme (« notre bateau a été plongé dans un épais brouillard. On n’y voyait pas à... »).

14 Sur le plan narratif interne, c'est-à-dire au niveau des récits proférés par les personnages de la diégèse, on peut encore relever l'insertion d'un récit minimal, et totalement banal, au premier plan, celui que raconte l'employé noir à Sammy dans les cases 1 et 2 ( « un jour j'y glissé et j'y vu --- des étoiles pendant une semaine »). Dans cette planche, on peut également relever les traces du préjugé racial du clivage Blanc / Noir, manifesté dans la façon « approximative» dont parle le domestique noir8. 
Mais ce clivage est tourné en dérision par McCay avec la représentation, dans l'image, de l'opposition entre le blanc de la neige et de la couleur de peau des promeneurs et le noir des cendres et de la couleur de peau de l'employé, et surtout avec la transformation des Blancs en «Noirs» suite au nuage de cendres, provoqué par l'éternuement de Sammy, qui vient recouvrir les deux mondains !

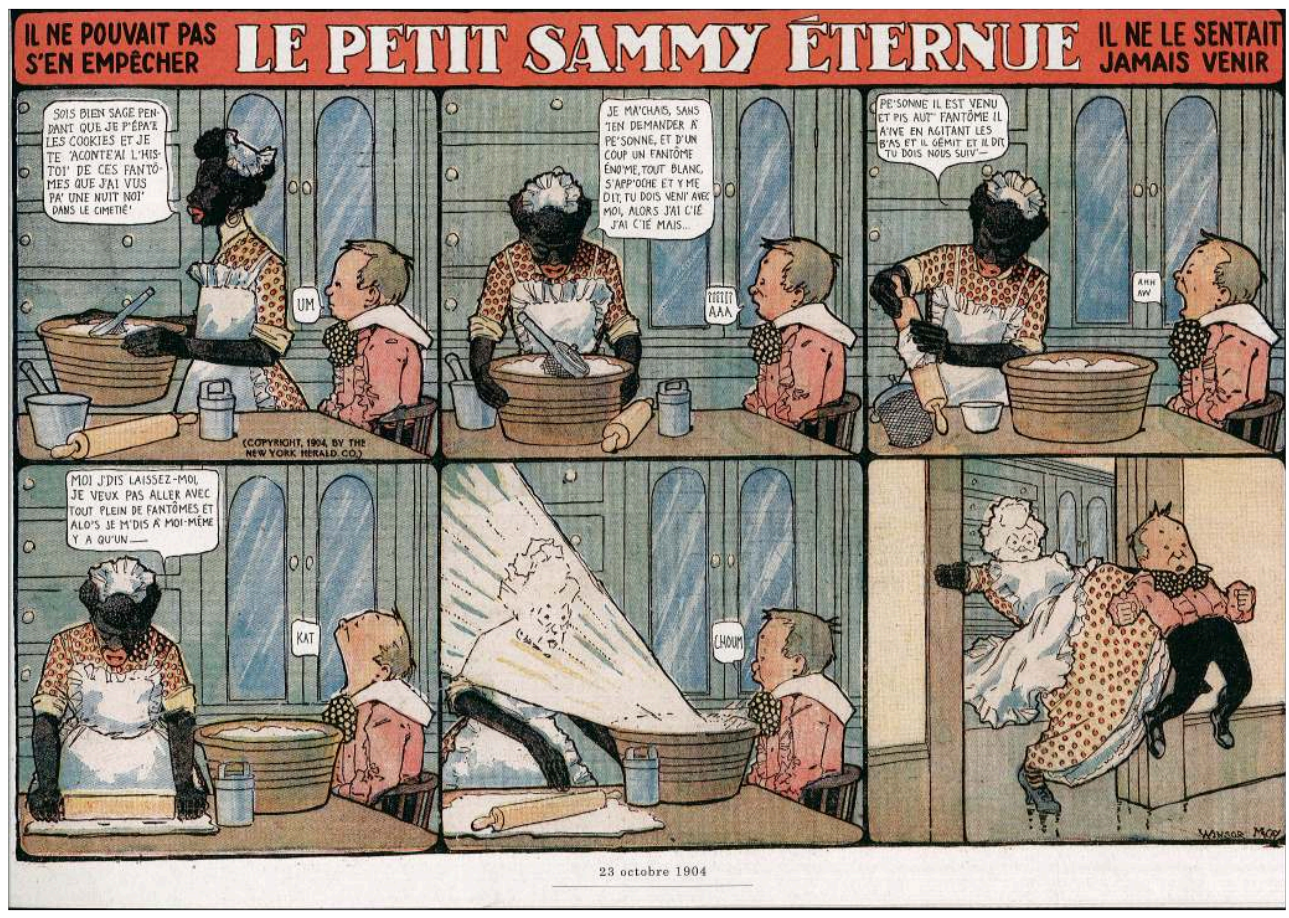

La planche 3 nous plonge dans le quotidien de Sammy, plus précisément dans sa cuisine, où une domestique est en train de préparer des biscuits. Dans la $1^{\mathrm{e}}$ case, la domestique propose un récit en échange du bon comportement de Sammy : «sois bien sage et je te raconterai ». Elle en annonce le thème (une histoire de fantômes, la nuit, dans un cimetière). Ce n'est pas banal et on peut imaginer que cela suscite de l'intérêt. Mais, comme d'habitude, Sammy ne réagit pas. Le récit de la cuisinière va donc être strictement monologal. Il se déroule dans la continuité des paroles d'une case à l'autre et il présente une structure narrative canonique : une situation initiale ( «je marchais sans rien demander à personne », un nœud «et d'un coup un fantôme énorme tout blanc s'approche... ». Au moment où, dans la case 4, la narratrice laisse entendre qu'elle a pensé à un dénouement possible lors de son aventure (« et alors je me dis à moi-même y a qu'un... »), son récit est interrompu par l'éternuement de Sammy, qui vient saboter non seulement sa performance narrative mais également le déroulement de son activité routinière (sur le mode du script « faire de la pâtisserie »). De façon paradoxale, l'interruption du récit verbal permet précisément à l'épisode de l'éternuement de trouver sa chute!

15 Si McCay, une fois encore, fait parler un personnage noir (selon le préjugé social et racial) dans une langue approximative, tout comme dans la planche précédente, il « rétablit » le clivage Blanc / Noir en recouvrant la cuisinière noire de farine blanche! On remarquera à ce propos que la transformation du noir en blanc est en quelque sorte annoncée dès la $1^{\mathrm{e}}$ case, les fantômes blancs par une nuit noire (du récit de la domestique noire) faisant écho à la farine blanche qu'elle manipule. 


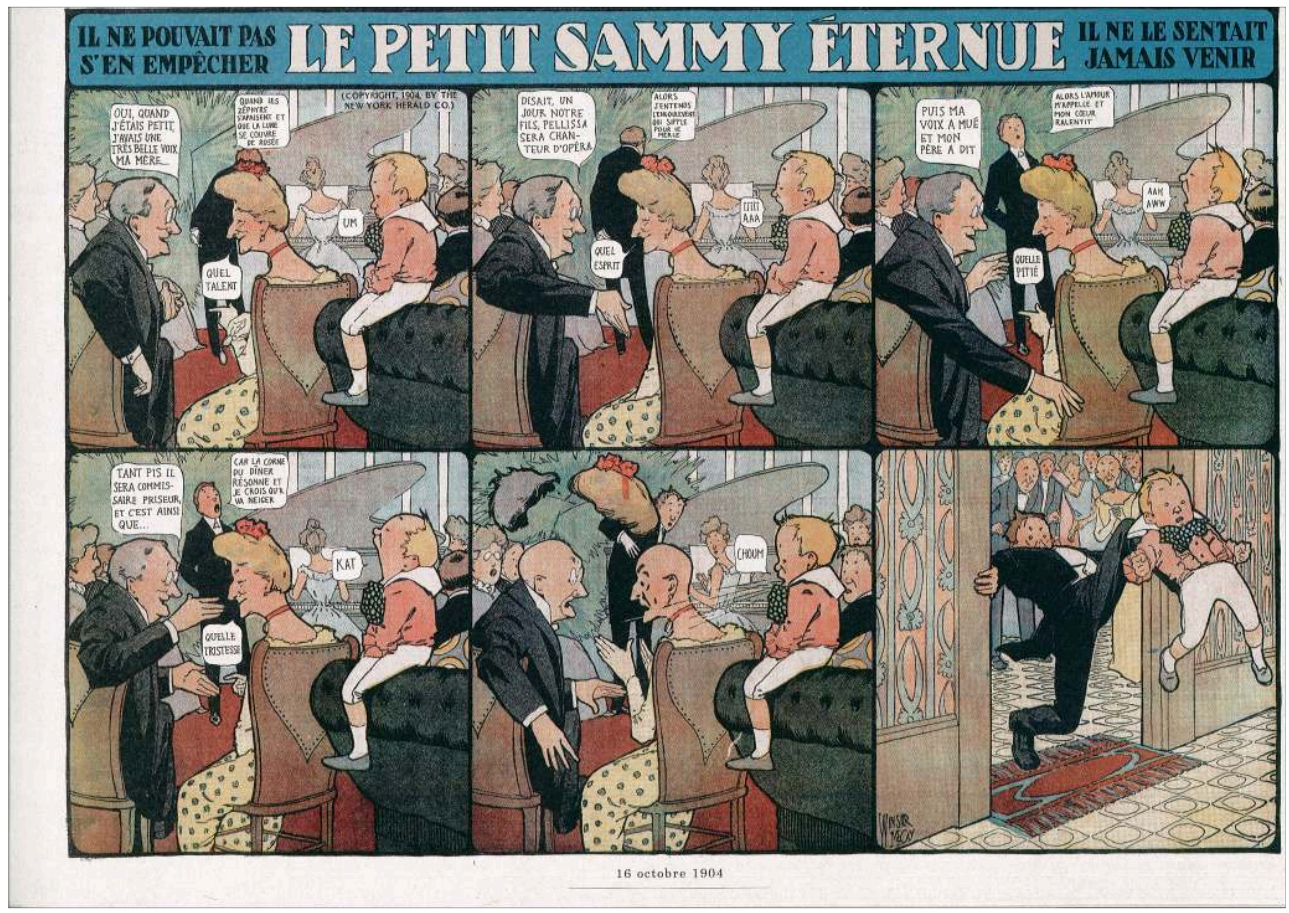

Cette dernière planche représente plusieurs activités qui se développent en parallèle lors d'une soirée mondaine à laquelle assiste Sammy : l'éternuement, une conversation privée et un récital de chant.

Tandis qu'à l'avant-plan se développe l'inévitable éternuement de Sammy, à peine en retrait, un vieil homme raconte à sa voisine son destin contrarié de chanteur d'opéra, ce qui n'est pas sans lien avec la situation de récital. Sa performance narrative est, ici aussi, interrompue par l'éternuement de Sammy. Quant à la scène de l'arrière-plan, elle peut passer relativement inaperçue à la première lecture. Il s'agit du récital de chant. Le chant constitue le fond sonore des quatre premières cases et on remarque que le contenu de ses paroles est totalement «plat» et non intrigant. Il relate une suite chronologique d'événements tous plus anodins les uns que les autres. Mais on peut noter qu'à la case 4 , le texte du chanteur semble annoncer le type de perturbation que va apporter l'éternuement de Sammy en affirmant « je crois qu'il va neiger ». De fait, ce seront les perruques qui sembleront tomber du ciel. Si les paroles du chanteur semblent pouvoir être ignorées dans un premier temps, le fait que ce dernier soit le seul personnage mobile (il change de posture entre la case 1 et la case 5 et c'est lui qui administre le coup de pied aux fesses de Sammy dans la dernière case) incite peut-être quand même le lecteur à aller voir de plus près le contenu des phylactères le concernant.

Dans cette planche, la performance narrative est encore une fois ridiculisée. Le récit du vieil homme est ponctué d'évaluations excessives de sa voisine (« quel talent !»; « quel esprit »; " quelle pitié »; "quelle tristesse ») alors qu'il est totalement banal et dénué d'intérêt. De plus, on assiste à une sorte de cacophonie avec une superposition des voix (les onomatopées de Sammy, les voix du vieil homme et de sa voisine, la voix du chanteur). Même dans les paroles de la chanson, il est question d'un engoulevent qui siffle et d'une corne qui résonne, éléments qui viennent ajouter leurs voix virtuelles au vacarme ambiant. 


\section{Conclusion}

18 Je conclurai sur un bref constat. On peut bien sûr lire les planches de la série Sammy Sneeze comme un simple divertissement, comme un objet commercial dont l'énorme succès devait permettre au journal qui l'hébergeait d'attirer le lectorat. D'ailleurs, le désintérêt général des chercheurs pour cette série tendrait à prouver qu'ils n'ont vu derrière la réitération du même gag au fil des semaines uniquement cet aspect récréatif et économique. En revanche, si l'on va voir « derrière » le gag, on constate que cette série a une dimension " politique » évidente puisque McCay glisse, sous les apparences cocasses de l'éternuement de Sammy, une critique sociale du mode de vie de ses contemporains, de leurs activités routinières et de leurs échanges verbaux vides de sens.

En conclusion, gardons-nous de cliver et de «pré-juger » les productions narratives en fonction de leur succès commercial...

\section{BIBLIOGRAPHY}

Barthes, R. (1970), S/Z, Paris : Editions du Seuil.

Baroni, R. (2007), La Tension narrative. Suspense, curiosité et surprise, Paris : Editions du Seuil.

Baroni, R. (2013), « La tension narrative à travers les genres : enjeux éthiques et esthétiques du suspense », Marti, M. \& Pélissier, N. (dirs), Tension narrative et storytelling, Paris : L'Harmattan, 17-35.

Boillat, A. (2012), « Le récit minimal en bande dessinée : l'histoire constamment réitérée d'un éternuement dans la série Little Sammy Sneeze de Winsor McCay ", Bedrane, S., Revaz, F. \& Viegnes, M. (éds), Le Récit minimal, Paris : Presses Sorbonne Nouvelle, 103-117.

Boillat, A. \& Revaz, F. (2016), «Intrigue, Suspense, and Sequentiality in Comic Strips : Reading Little Sammy Sneeze », Baroni, R. \& Revaz, F. (éds), Narrative Sequence in Contemporary Narratology, Columbus : The Ohio State University Press, 107-129.

Peeters, B. (1990), « Un inventeur du dimanche », Groensteen, T. (éd.), Little Nemo au pays de Winsor McCay, Toulouse : Editions du Milan/CNBDI.

\section{NOTES}

1. A la suite de Baroni (2013), je rappelle l'anathème lancé contre l'intrigue « commerciale » et le suspense que Barthes associait « aux habitudes commerciales et idéologiques de notre société qui recommande de "jeter" l'histoire une fois qu'elle a été consommée (“dévorée"), pour que l'on puisse alors passer à une autre histoire, acheter un autre livre. » (Barthes, $1970: 20$ ).

2. Cette série a peu été étudiée (à la différence de Little Nemo in Slumberland, du même auteur). En effet, elle a toujours été considérée comme mineure dans l'œuvre de McCay, son dispositif étant la répétition inlassable, sur toute la durée de la série, du même gag, dans le même gaufrier, alors 
que les planches hebdomadaires de Little Nemo proposent des mises en page variées, preuves d'une inventivité et d'une aisance technique extraordinaires.

3. Le développement temporel de l'éternuement s'effectue par le biais d'une référence au modèle chronophotographique de la représentation du mouvement. Il renvoie à certaines expériences de décomposition et de restitution du mouvement par la prise de vue photographique.

4. L'avant-dernière vignette dédiée à la monstration des effets de l'éternuement s'inscrit dans ce que les théoriciens du cinéma des premiers temps ont appelé une logique " attractionnelle ». Ce type de gags est omniprésent dans les films comiques de la période 1895-1910; ils reposent sur la mise en place d'une action aux suites totalement prévisibles (si ce n'est dans leur ampleur).

5. Sur l'aspect minimal de la série, lire Boillat 2012.

6. Dans la version originale anglaise : « He just simply couldn't stop it », « He never knew when it was coming ".

7. Sur la dimension séquentielle, lire Boillat et Revaz 2016.

8. La traduction française reprend les approximations de la prononciation et de la syntaxe mises en évidence par McCay en anglais.

\section{ABSTRACTS}

The aim of this article is to show that the series of comic strips Little Sammy Sneeze has the characteristic of being both "recreational" and "political". Under the guise of an entertaining narrative sketch giving, each week, a new occurrence of devastating little Sammy's sneeze, McCay takes a critical and satirical look, not only on the serial production mode of the contemporary comics, but also on the way of life of the American middle class.

This example will make it possible to examine the relevance of the dichotomous distinction between the fictive literature where its sole purpose would be to provide the reader with entertaining adventures and the factual literature that would have a monopoly on social and political questions.

L'objectif de cet article est de montrer que la série de bandes dessinées Little Sammy Sneeze a pour particularité d'être à la fois « récréative » et " politique ». Sous l'apparence d'un schéma narratif divertissant donnant à voir, chaque semaine, une nouvelle occurrence de l'éternuement dévastateur du petit Sammy, McCay pose un regard critique et satirique, non seulement sur le mode de production sériel des bandes dessinées de son époque, mais également sur le mode de vie de la classe moyenne américaine.

Cet exemple permettra ainsi d'interroger la pertinence de la distinction dichotomique entre, d'une part, une littérature dite "d'imagination» dont le seul but serait de proposer au lecteur des aventures divertissantes et, d'autre part, une littérature «factuelle » qui aurait le monopole du questionnement social et politique. 
INDEX

Chronological index: XXe siècle

Keywords: comic strips, intrigue, parody, satire, series, stops comics

Mots-clés: bandes dessinées, intrigue, parodie, satire, série, stop comics

Geographical index: Etats-Unis

\section{AUTHOR}

\section{FRANÇOISE REVAZ}

Université de Fribourg, Suisse

Françoise Revaz est professeure de linguistique française à la Faculté des Lettres de l'Université de Fribourg. Spécialiste en narratologie, elle a fondé, avec R. Baroni, le Réseau Romand de Narratologie. Auteure de L'analyse des récits, en collaboration avec J.-M. Adam (Seuil, 1996), des Textes d'action (Klincksieck, 1997) et de Introduction à la narratologie. Action et narration (De Boeck \& Duculot, 2009), elle a dirigé plusieurs projets de recherche sur les formes et fonctions du récit dans divers genres de discours (littérature, historiographie, presse écrite et bande dessinée). Actuellement, ses travaux sont centrés sur les types de découpage dans les narrations sérielles. 